\title{
Measurement of Multivalued Response Curves of a Strongly Nonlinear System by Exploiting Exciter Dynamics
}

\author{
Genbei Zhang ${ }^{\mathrm{a}}$, Chaoping Zang ${ }^{\mathrm{a}^{*}}$, Michael I. Friswell ${ }^{\mathrm{b}}$ \\ ajiangsu Province Key Laboratory of Aerospace Power System, Nanjing University of \\ Aeronautics and Astronautics, Nanjing, 210016, P R China \\ ${ }^{\mathrm{b}}$ College of Engineering, Swansea University, Swansea SA1 8EN, United Kingdom
}

\begin{abstract}
A strongly nonlinear system often has multiple solutions under harmonic excitation. However, measuring all of these multiple responses in structural dynamics is challenging because often one solution is unstable and difficult to obtain. The standard stepped sine approach is to fix the harmonic excitation force amplitude, and step the excitation frequency up or down. This leads to the well-known jump phenomenon, and captures at most two stable solutions. Alternatively, the excitation frequency can be fixed and the amplitude swept up or down, although this also leads to jumps in the response. Recently, experimental continuation methods have successfully measured all solutions, including the unstable solutions, via active control. This paper takes a different approach and exploits the dynamics of the electromagnetic exciter to both stabilize the unstable solution, and also to track the solutions continuously, without any jumps. This is achieved by monotonically increasing or decreasing the voltage applied to the exciter at a fixed frequency, and using the force drop-out phenomenon through the resonance to control the force applied to the structure. In these tests, the input voltage then defines the continuation parameter, rather than force amplitude or frequency in the standard tests. The obvious advantage of this method is that there is no feedback control of the excitation and it is easy to implement. A strongly nonlinear single degree of freedom system is used to demonstrate this method.
\end{abstract}

\section{Keywords}

Force drop-out phenomena, multivalued response curves, continuation, nonlinear system 


\section{Highlights:}

- The force drop-out phenomenon at resonance in strongly nonlinear systems is exploited for fixed frequency sinusoidal tests.

- The input voltage of electromagnetic exciter is considered as a natural continuation parameter for strongly nonlinear systems.

- A fixed frequency test is proposed to measure multivalued response curves of strongly nonlinear systems without feedback control of the excitation.

\section{Introduction}

Engineering structures are often treated as linear structures, although in many cases this is clearly an approximation and simplification. These structures often contain nonlinearities, and typical sources of nonlinearities in structural dynamics include geometric nonlinearity, material nonlinearity, inertial nonlinearity, damping dissipation and nonlinearities caused by boundary conditions [1]. Clearly, the relationship between the steady-state harmonic excitation and the periodic response is linear for a linear system, or approximately so for low amplitude excitation of nonlinear structures. For a strongly nonlinear system, jump phenomena may occur because of the multivalued nature of the response curves [2]. Such strongly nonlinear behavior can be found in many nonlinear structures, such as beams with local nonlinearities [4], turbine blades with nonlinear contacts [5], bolted joint structures [6], complex aerospace structures [7] and even in micro-electromechanical systems (MEMS) [8]. The multiple solutions of nonlinear systems are a clear difference between linear and strongly nonlinear oscillations[3].

The multivalued response of strongly nonlinear systems has been discussed in detail [2] in the theoretical study of nonlinear vibration. In recent years, different numerical methods and software toolkits have been developed, which can be used to solve for the periodic solutions of strongly nonlinear systems [9], including the shooting method based on Newmark time integration [10], orthogonal collocation methods and the harmonic balance method in the frequency domain [11]. These methods are mainly based on a continuation procedure [12]. The orthogonal collocation methods have developed a variety of software toolkits such as COLSYS, AUTO, MATCONT and COCO [9]. The harmonic balance method can also be used in the vibration analysis of large-scale nonlinear finite element models [5][13]. However, significant difficulties and challenges still exist in the measurement of the multivalued response curve of a strongly nonlinear system.

In modal testing, the electromagnetic shaker is the most common type of exciter in which the supplied excitation signals can be continuous, such as sinusoidal and random, or transient, such 
as the pulse and chirp. These different excitation signals have their own advantages and disadvantages [14]. Generally, sinusoidal excitation is an important periodic excitation used to investigate the nonlinear dynamic characteristics of structures because the frequency and amplitude can be accurately controlled independently. However, the electromagnetic shaker is not an ideal source of energy, and the actual amplitude of the excitation force will change due to the vibration of the structure, especially near resonance. A force drop-out phenomenon can happen in the swept sine test of a linear structure or a nonlinear structure [15][16] and this has been discussed by several scholars [17][18][19]. Furthermore the magnetic field of the exciter is generally considered to be nonlinear and can be assumed to be linear only under small vibration conditions. As the armature moves in the magnetic field the large vibration near resonance causes the interaction force between the exciter and the structure to become very small [14]. Generally speaking, if the structure under test is linear, the influence of this dropout phenomena can be ignored in the calculation of the frequency response function (FRF) measurement because the FRFs are independent of excitation amplitude. However, this force drop-out cannot be ignored for nonlinear structures [20].

In the theoretical analysis of nonlinear systems the input force is commonly assumed to be a simple harmonic excitation with a constant force level. In order to compare the theoretical and experimental results, the actual excitation force in experiments is often tuned to ensure a constant amplitude harmonic excitation force. Controlled-level vibration tests (CLV) are developed to keep the force level constant at resonance using nonlinear force control algorithms [14][15]. This method has been implemented in many commercial products and is widely used. Example experiments where the force amplitude of the electromagnetic shaker is kept constant have been widely reported [4][9][21]. Often there are three possible steady-state periodic responses in the frequency response curve of a strongly nonlinear system near resonance, which are usually termed the higher, middle and lower branches, depending on the response amplitude. The actual response obtained will depend on the initial conditions and the phase relationship between the response and the force will be different. The middle branch is often unstable and cannot be observed in practice [22]. The CLV test method can obtain the higher and lower solution branches of strongly nonlinear systems through forward and backward swept sine tests, but cannot obtain the middle branch. The continuous response curve cannot be measured in a CLV test, which leads to the occurrence of the jump phenomenon in experiments.

In order to measure the continuous frequency response curve of a strongly nonlinear system, the arc length continuation method in nonlinear numerical computation has been introduced into experimental tests as a control strategy and an intelligent nonlinear coupling analysis (INCA) method was proposed by Ferreira et al. [14][23]. Although this technique gives good results, it is difficult to choose the control parameters in the experiments to obtain the derivative from the finite difference and the appropriate radius of the constraint equation [23]. Remarkably, 
the unstable branch is measured by this method. The explanation from Ferreira is that although the nonlinear structure itself has an unstable response, the structure composed of the shaker plus the nonlinear structure is stable [14]; the stablizing effect of the exciter will also be used in the method proposed in this paper.

Recently, a control-based continuation method (CBC) for experimental bifurcation analysis has been developed by Sieber et al. [24][25]. This method can be used to measure the multivalued frequency response curves of strongly nonlinear systems. The detailed development of this method can be found in reference [26]. Barton et al. [27] applied this method to a nonlinear energy harvester based on the idea of numerical continuation to perform pseudo-arc length continuation through feedback control to measure the nonlinear frequency response curve and demonstrated good results. Furthermore, they kept the forcing frequency fixed rather than the forcing amplitude to measure the response curve for each given frequency [28][29]. Because the forcing frequency doesn't need to be controlled, the path-following techniques used in CBC could be simplified, resulting in a significant speed up of the method. In addition, an indirect method for the synthesis of nonlinear frequency responses near an isolated resonance was developed by Peter et al. [30]. This method is based on standard linear and nonlinear modal testing using a variant of the phase resonance method in order to avoid the difficulties related to the nonlinear frequency response function measurements. In 1979, Nayfeh and Mook proposed two hypotheses on the experimental measurement of jump phenomena of a nonlinear system [2]. One is that if the experiment is performed in which the amplitude of the excitation is kept constant, while the frequency of the excitation is very slowly swept up or down through the resonance (constant force tests), and a jump phenomenon will be observed. This hypothesis has been verified in many experiments [4][9][21]. The alternative approach is to perform the experiment with the frequency of excitation held fixed, while the amplitude of the excitation is varied slowly (fixed frequency tests), and a similar jump phenomenon may be observed. However, this type of test is rarely undertaken and hence rarely reported.

In this paper, the force drop-out phenomenon for electromagnetic exciters in a strongly nonlinear system is exploited in fixed frequency tests. When the input voltage of the shaker increases slowly in a fixed frequency test, the amplitude of the fundamental frequency component of the excitation force to the structure will not increase monotonically, but increases at first, then decreases, and finally increases at frequencies near resonance; in contrast the response amplitude keeps increasing. During this sweep, there are multiple (usually three) response amplitudes corresponding to the same excitation amplitude. This phenomenon only occurs in strongly nonlinear systems and the effect is different from the force drop-out near resonance for a linear structure. Thus the input voltage of the electromagnetic shaker can be considered as a natural continuation parameter in fixed frequency tests. Since the input voltage varies monotonically (for example, increases linearly), the multivalued response curve can be 
guided smoothly through the turning point and traverse the bifurcation point of the solution. Such a continuation parameter has physical significance in practice and can be controlled directly. Therefore, the test process will be greatly simplified and is easy to be implement. Although the arc length and the pseudo arc length in the previously introduced INCA [14][23] and CBC methods [24][25] are also used as the continuation parameters, they have geometrical rather than physical meaning and cannot be controlled directly.

The structure of this paper is as follows. In Section 2, force drop-out phenomena are introduced through a weakly and a strongly nonlinear system. In particular, a new force dropout phenomenon peculiar to the strongly nonlinear system found in fixed frequency sinusoidal tests is discussed in detail. In Section 3, the relationship between the excitation and input voltage, and the relationship between the response and input voltage are discussed respectively. It is found that the input voltage can be considered as an ideal continuation parameter. In Section 4, an experimental measurement method of multivalued responses of a strongly nonlinear system is proposed based on the force drop-out phenomena, taking the input voltage as a continuation parameter. Measured results are given in Section 5, where influence of excitation on the measured multivalued responses are discussed. Section 6 draws the conclusions of this paper.

\section{Response Measurement of Nonlinear Structures}

Stepped or swept sine tests are commonly used for nonlinear systems as they are easy to perform and emphasize any nonlinear behavior in the structure. Furthermore, such tests are often used in the analysis of nonlinear systems and so the measurements can be directly compared to simulated results. There are two types of sine test commonly used for structures: the fixed force test and the fixed frequency test, as shown in Fig. 1. In the fixed force test, shown in Fig. 1(a), the harmonic input force amplitude is fixed and the excitation frequency is stepped up or down in small increments (or slowly swept) and the response measured at the excitation frequency. At each frequency a delay before the measurement is taken allows the response of the structure to reach the steady state. During the step from one frequency to the next, the force is continuous to minimize the transients and ensure the response stays on the solution branch of interest. The test is then repeated for a different force amplitude. In contrast, the excitation frequency may be fixed and the force amplitude slowly increased or decreased, as shown in Fig. 1 (b).

Both the fixed force amplitude and the fixed excitation frequency need feedback control on the force since the output from the signal analyzer will be a voltage input to the shaker amplifier. The force drop-out phenomenon, discussed in depth in the next section, means that the force 
will reduce at resonance for a fixed voltage. Most commercial analyzers used for stepped sine testing will include facilities for this force control, although this does significantly increase the length of the test. However, the frequency response functions obtained using this approach can be compared directly to the simulated results. The other issue is that the coupling between the nonlinear structure and the exciter will cause the excitation force to contain harmonics of the fundamental excitation frequency, even though the voltage input is a pure sinusoid. Including harmonics of the fundamental frequency in the voltage input can be used, with suitable feedback control, to eliminate the harmonics in the force and provide a purely sinusoidal force (see for example Shaw et al. [33]).

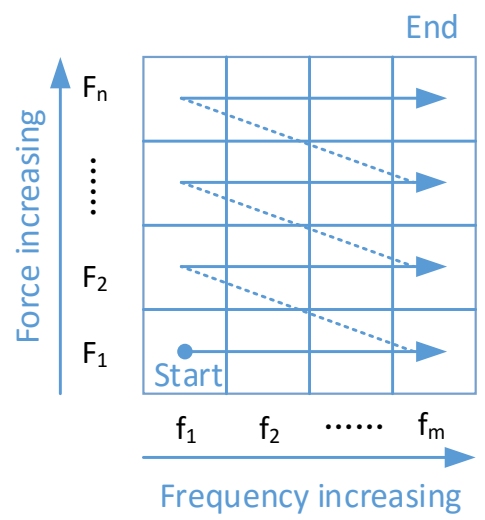

(a) Fixed force test

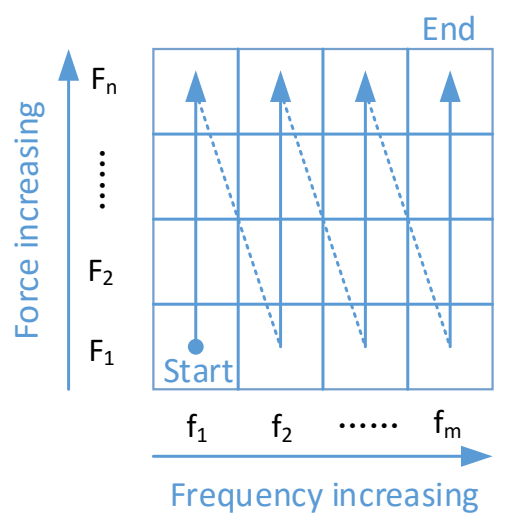

(b) Fixed frequency test

Fig. 1 The two common sine test methods

The response obtained from the fixed force amplitude and fixed frequency tests may be demonstrated by the simulated results of a single degree of freedom (SDOF) system with a cubic stiffness. The theoretical analysis can generate the multivalued response curve of a nonlinear system based on fixed force amplitude or fixed excitation frequency [2]. These simulated multivalued responses from a typical SDOF system are shown in Fig. 2. The green curves give the upper and lower stable branches for the fixed force amplitude test. Starting at low frequency for a stepped frequency test, the response would follow the upper branch until the resonance point, where it would jump to the lower branch at higher frequencies. Similarly starting at a high frequency the response would follow the lower branch until the turning point, where it would jump to the upper branch. The middle solution, give in magenta, is unstable and cannot be reached by standard stepped sine testing. The blue curve gives the upper and lower branches for the fixed frequency test, where the force is either slowly increased or decreased. Jumps will also occur in the corresponding responses, and the unstable middle branch, given in magenta, again cannot be reached. 
This simple example highlights the two issues in obtaining the full FRF curve for nonlinear systems. Using force amplitude or excitation frequency as the continuation parameter is unable to negotiate the turning points, and hence cannot obtain the middle branch of the solution. Also, the middle branch is often unstable and hence cannot be obtained in a standard test. Using the force drop-out phenomena for electrodynamic exciters is able to solve both of these problems.

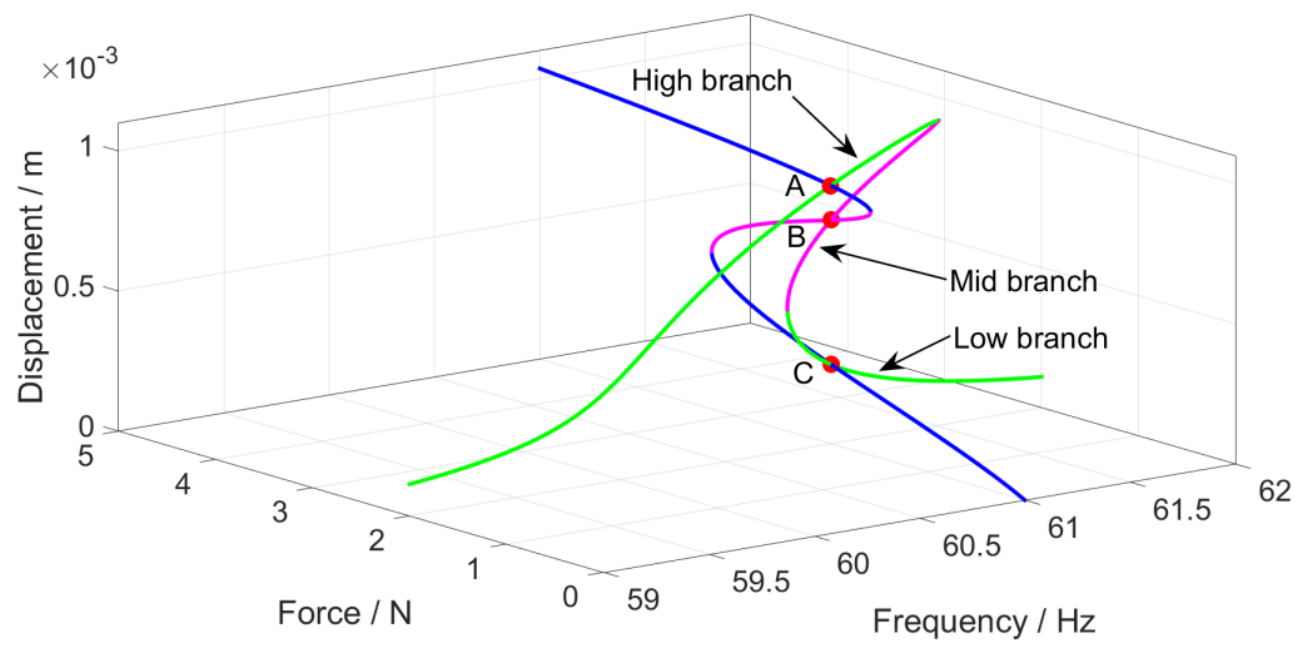

Fig. 2 Multivalued response curves of a cubic SDOF system

\section{The Force Drop-out Phenomena in Nonlinear Systems}

In stepped or swept sine tests using an electromagnetic shaker without feedback control, the force drop-out phenomenon will occur near resonance, for both linear or nonlinear structures. However, there is a significant difference in the effect of the force drop-out phenomena between linear or weakly nonlinear systems and strongly nonlinear systems. In order to illustrate the difference, sine tests at different excitation voltage levels have been performed on a weakly nonlinear system and a strongly nonlinear system. Note that these systems are different and are only used here to demonstrate the different effects due to the nonlinearity.

\subsection{Force drop-out phenomena in a weakly nonlinear system}

A weakly nonlinear system is shown in Fig. 3, which consists of a frame structure with bolted joints. There are three steel plates, which may be considered as three lumped masses, and the four aluminum beams are equivalent to three springs in this structure. The size of each steel plate is $150 \mathrm{~mm} \times 100 \mathrm{~mm} \times 10 \mathrm{~mm}$ with mass density of $7820 \mathrm{~kg} / \mathrm{m}^{3}$, so each plate has a mass of about $1.173 \mathrm{~kg}$. The size of each aluminum beam is $220 \mathrm{~mm} \times 30 \mathrm{~mm} \times 2 \mathrm{~mm}$. Four rubber rings 
are installed between the four aluminum beams and the base plate to introduce unknown nonlinear stiffness and damping behavior. This structure represents a three DOF weakly nonlinear system for the lateral vibration and it is excited by one ET-140 electrodynamic shaker from Labworks Inc. More details and experimental results for this structure are given in [31].

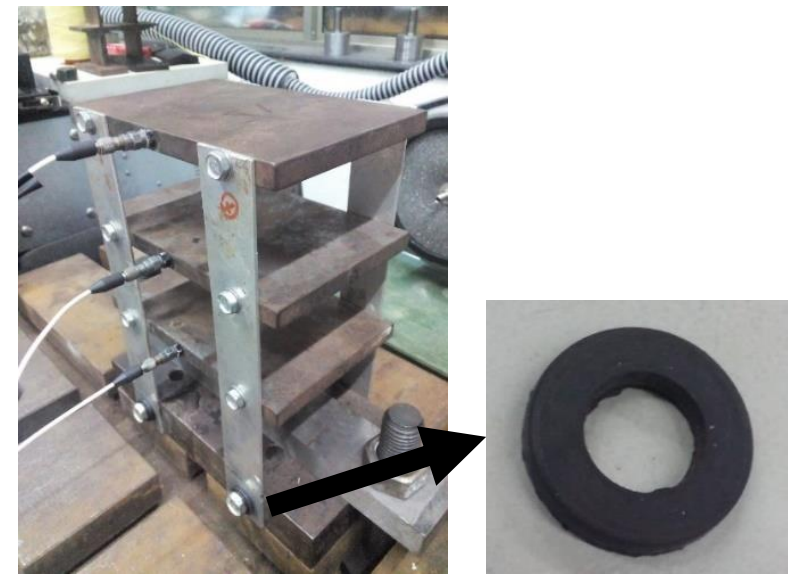

(a) Experimental setup

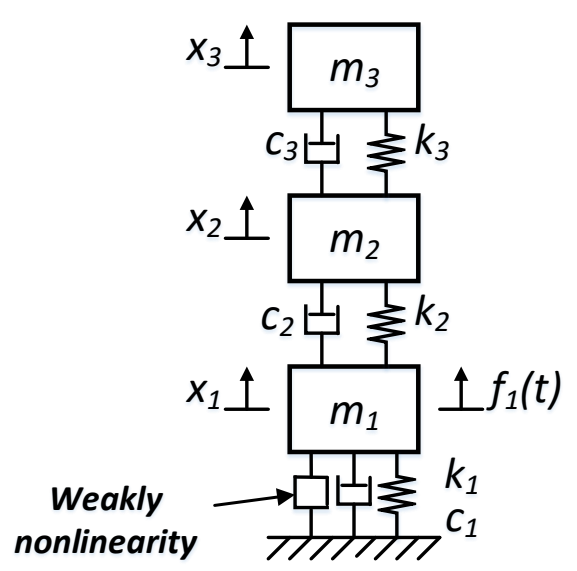

(b) Dynamic model

Fig. 3 The three DOF weakly nonlinear system

In the stepped sine tests, an electromagnetic shaker is used to excite the first mass; the force input is directly measured and the responses are measured using accelerometers. The frequency range is $20 \mathrm{~Hz}$ to $27 \mathrm{~Hz}$ (covering the first resonance), and the frequency step size is $0.1 \mathrm{~Hz}$. The input voltage range of the signal generator is $0.05 \mathrm{~V}$ to $1 \mathrm{~V}$, and the step size is $0.05 \mathrm{~V}$. A delay of 3 seconds after each test ensures that the response transients have decayed.

When the input voltage is $1 \mathrm{~V}$, the first harmonic of the force and the fundamental frequency response functions are shown in Fig. 4. The force drop-out clearly occurs near resonance, since the excitation force is $13.4 \mathrm{~N}$ at $20 \mathrm{~Hz}$, and then drops with increasing frequency. At resonance (about $23 \mathrm{~Hz}$ ) the force is reduced to $6.4 \mathrm{~N}$, and then increases for high frequencies. This kind of force drop-out phenomenon is well known in stepped and swept sine tests, and also occurs with broadband excitation. 


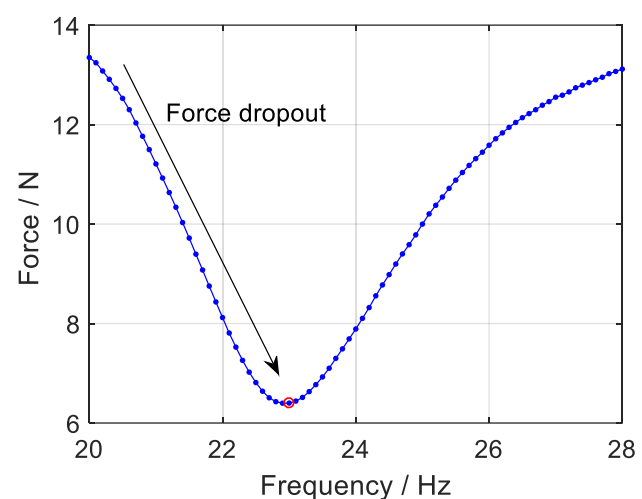

(a) Force drop-out phenomenon

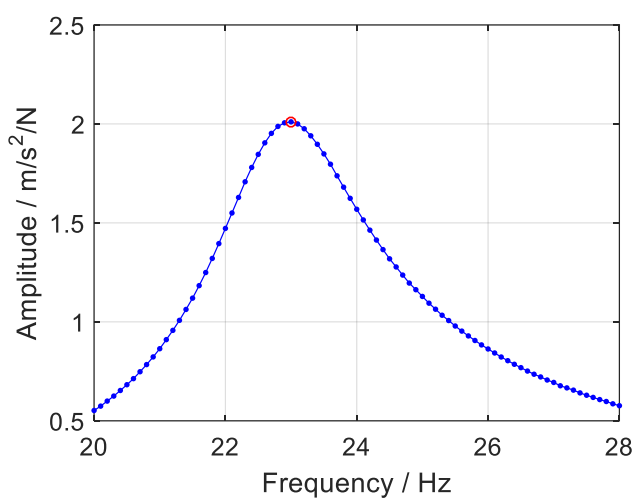

(b) The acceleration FRF

Fig. 4 The force drop-out phenomenon for the three DOF structure

The results of a group of 20 tests for different excitation voltages are shown in Fig. 5. The resonance frequency clearly decreases with an increase in the excitation voltage because of the nonlinear softening stiffness. The shift in the resonance frequency also corresponds to the minimum force during the drop-out. Note that in a linear system the resonance frequency would not change with excitation amplitude.

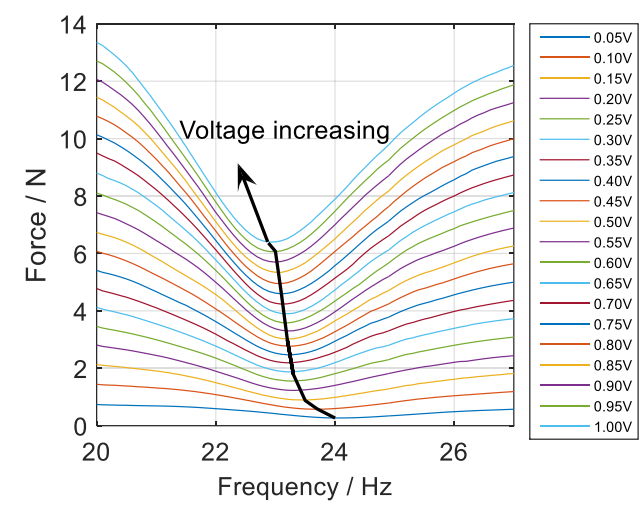

(a) Force drop-out phenomenon

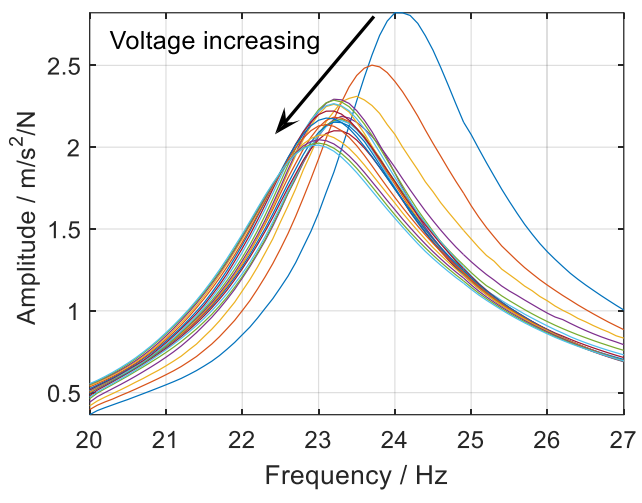

(b) The acceleration FRF

Fig. 5 Force drop-out phenomenon in the weakly nonlinear system

\subsection{Force drop-out phenomena in a strongly nonlinear system}

A strongly nonlinear system is shown in Fig. 6. A square steel block and two thin plates are assembled and fixed on a square frame by screw connections, while the frame is fixed to the foundation. Only the lateral vibration of the first mode is considered. The elastic recovery force provided by the thin steel plates is close to a cubic nonlinearity. This structure represents a 
SDOF system with cubic nonlinearity and it is also excited by one ET-140 electrodynamic shaker from Labworks Inc. A more detailed introduction to this kind of nonlinearity can be found in [21] and [33].

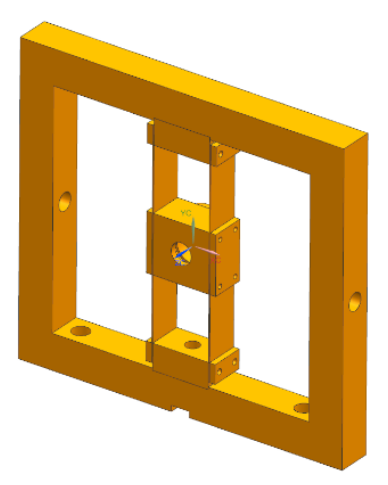

(a) 3D model

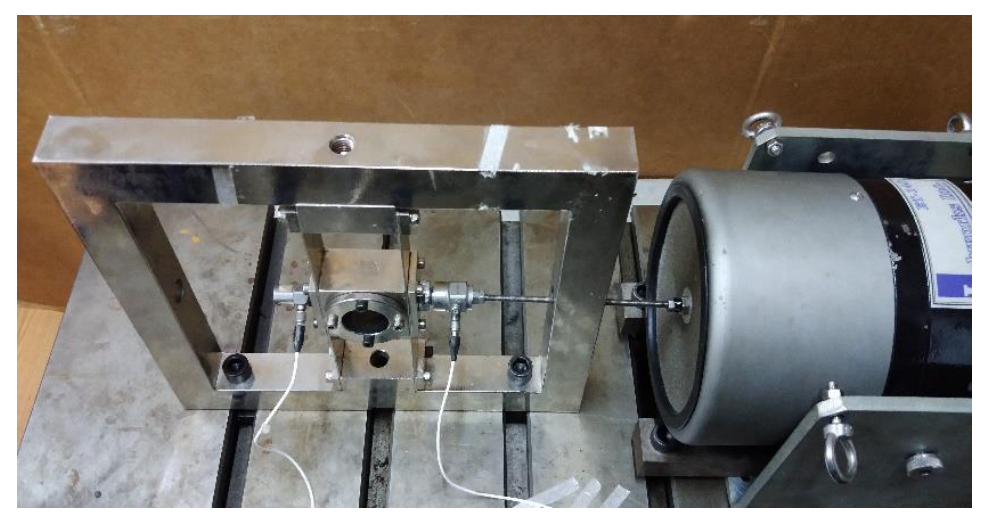

(b) Experimental setup

Fig. 6 A strongly nonlinear system representing a rotor bearing support

The frequency range for the experiments is selected as $59 \mathrm{~Hz}$ to $64 \mathrm{~Hz}$, which covers the resonance of the first mode and the frequency step size is $0.1 \mathrm{~Hz}$. The input voltage range of the signal generator is $0.05 \mathrm{~V}$ to $2 \mathrm{~V}$, and the step size is $0.05 \mathrm{~V}$. The results of a group of 40 tests at different voltage levels are shown in Fig. 7. Clearly there are strong force drop-outs near resonance and the shift of the resonance frequency corresponds to the minimum force. The resonance frequency increases with the excitation voltage, due to the hardening stiffness of the nonlinearity.

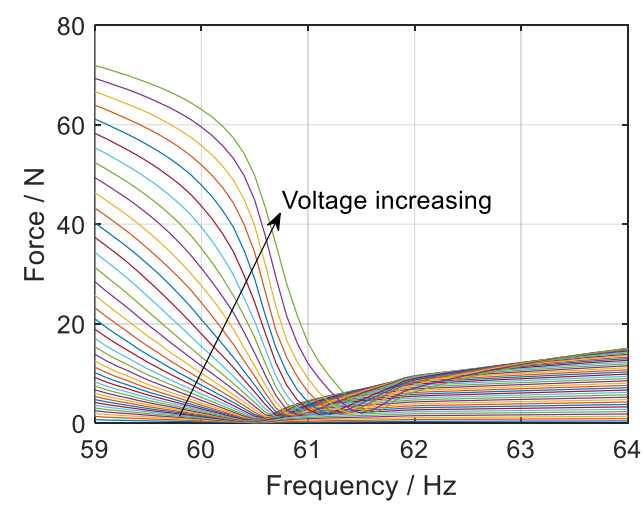

(a) Force drop-out phenomena

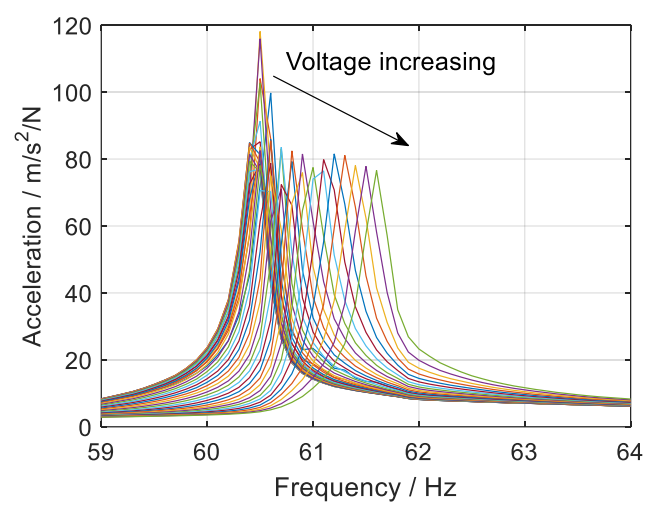

(b) The acceleration FRFs

Fig. 7 Force drop-out phenomenon in the strongly nonlinear system as the voltage increases from $0.05 \mathrm{~V}$ to $2.00 \mathrm{~V}$ with a step of $0.05 \mathrm{~V}$

In order to observe the force drop-out in more detail, the test curves for selected voltages and close to the resonance frequencies are shown in Fig. 8. In this case, the force curves at different 
voltages cross over each other, especially for higher voltages. Thus the excitation force amplitude may decrease for some frequencies near resonance as the input voltage increases.

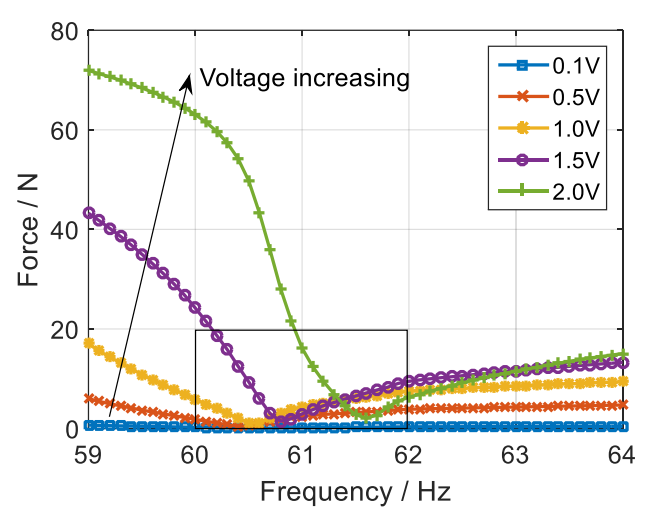

(a) Force drop-out for selected voltages

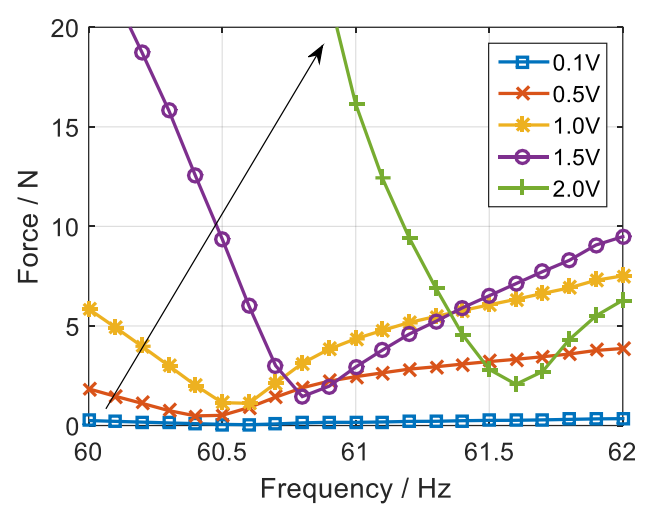

(b) Force drop-out from $60 \mathrm{~Hz}$ to $62 \mathrm{~Hz}$

Fig. 8 Force drop-out phenomenon in the strongly nonlinear system in detail

\subsection{Comparing weakly and strongly nonlinear systems}

By comparing the test results of force-drop phenomena in Sections 3.1 and 3.2, we can find their similarities and differences. The force drop-out will occur near resonance in stepped or swept sine tests, and the frequencies corresponding to the maximum force drop changes with the increase of the input voltage level to the electromagnetic shaker. The direction and degree of this shift depends on the form of the nonlinearity. The major difference is that the force drop curves of the strongly nonlinear system under different excitation levels will cross each other, while those for the weakly nonlinear system will not. In order to demonstrate this difference more clearly, the relationship between the force at a single frequency near resonance and the input voltage is shown in Fig. 9. Although the relationship between the force and voltage is no longer linear, it remains monotonic for the weakly nonlinear system, so that the excitation force amplitude increases with increasing input voltage, as shown in Fig. 9(a). However, this monotonicity does not occur at many frequencies near resonance for the strongly nonlinear system, as shown in Fig. 9(b). The force amplitude increases initially, then decreases, before increasing again. This is a new interpretation of the force drop-out phenomenon in fixed frequency tests. This force drop-out means that there are multiple input voltages corresponding to the same excitation amplitude, and this will be exploited in the tests described in detail in Section 4. In experiments, if this kind of force drop-out phenomenon occurs in a system, it shows that the system has a strongly nonlinear behavior with multiple responses and co-existing solutions. 


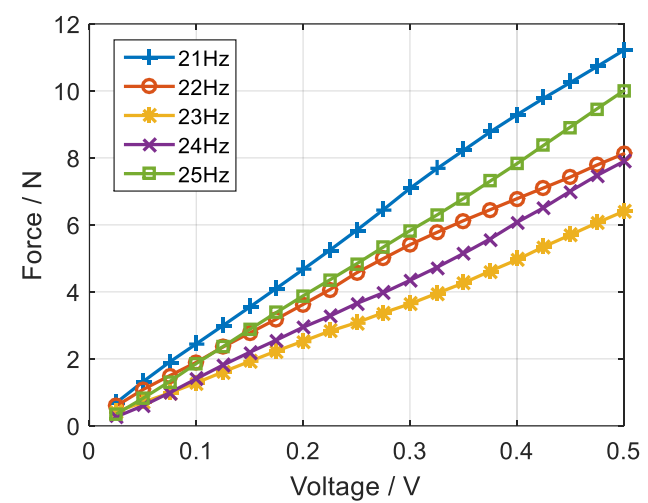

(a) Weakly nonlinear system

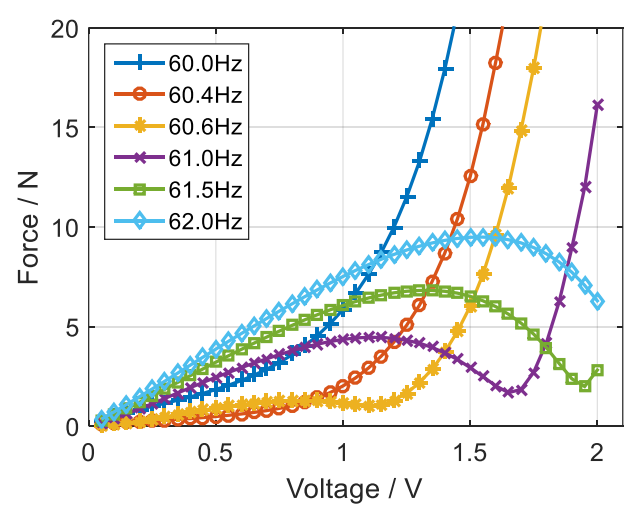

(b) Strongly nonlinear system

Fig. 9 The relationship between the force and voltage at fixed frequencies

\section{Measurement with input voltage as the continuation parameter}

From the view of continuation measurement, if we want to obtain a multivalued response curve, we need to control the excitation frequency drop-out in the constant force test (see the green curve in Fig. 2), or control the excitation amplitude drop-out in the fixed frequency test (see the blue curve in Fig. 2) through the continuation parameter which will guide the response curve through the turning point smoothly. In experiments, the electromagnetic shaker has dynamic characteristics that couple with the structure and cause a relationship between the excitation frequency and amplitude. When the excitation frequency is fixed, the excitation force amplitude will be affected by the vibration of the structure, as shown in Section 3. Therefore, one option is to control the excitation force amplitude through the resonance to keep the excitation amplitude constant, which would require an increase in the input voltage through resonance. However, this force control would mean that the unstable middle branch response cannot be obtained and jump phenomenon would occur. In the fixed frequency test, only one parameter that determines the excitation amplitude needs to be controlled to realize the measurement of multivalued response curves. Therefore, the force drop-out in the fixed frequency test is exploited here to measure the multivalued response curve of a strongly nonlinear system.

Generally, the measurement of the multivalued response curve requires the selection of an appropriate continuation parameter. An ideal continuation parameter can uniquely identify each solution on the branch and traverse the whole solution through monotonic variation of the parameter [12]. Generally, the continuation parameters used, such arc length or pseudo arc length, only have a geometrical rather than a physical meaning, and cannot be controlled directly in experiments. If a physical continuation parameter exists, then this would lead to a much simpler test. Here, the input voltage of the electromagnetic shaker is proposed as a 
suitable physical continuation parameter, and in the fixed frequency test the force drop-out is used to indirectly control the excitation force.

The strongly nonlinear example given in Section 3.2 is used to demonstrate the approach at the fixed excitation frequency of $61.2 \mathrm{~Hz}$, which is close to the first resonance, and the variation of the first harmonic of the excitation and response with the input voltage is measured. The linear increase of the input voltage to the electromagnetic shaker during the test is shown in Fig. 10. The voltage range is from $0.05 \mathrm{~V}$ to $2 \mathrm{~V}$ and the step size is $0.05 \mathrm{~V}$. A total of 40 voltage levels are used and hence 40 single frequency sinusoidal tests are performed.

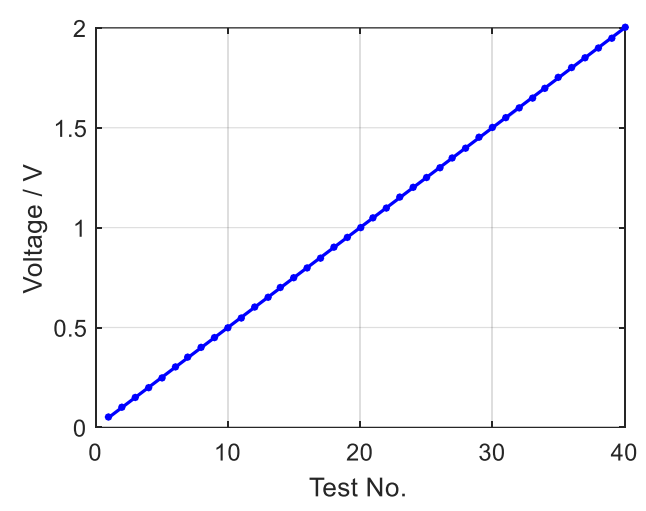

Fig. 10 Linear increase of the input voltage in the fixed frequency tests

The relationship between the first harmonic of the excitation amplitude and the voltage is shown in Fig. 11. With the increase of input voltage, the change of excitation amplitude can be divided into three stages. Between points A and D, the excitation amplitude increases - initially the trend is approximately linear (because the coupling to the shaker dynamics is low), but the rate of increase reduces and reaches a maximum at $\mathrm{D}$. From point $\mathrm{D}$ to point $\mathrm{F}$, the force dropout phenomenon occurs, where the force amplitude decreases and reaches a minimum at $\mathrm{F}$. Finally, the force amplitude increases again after point $\mathrm{F}$.

The force drop-out between points $\mathrm{D}$ and $\mathrm{F}$, forms a multivalued region in the rectangle given by B, K, H and J. Here, a many to one mapping is formed between voltage and force, although the force is a single valued function of voltage, and hence there is a unique excitation amplitude corresponding to each voltage. In contrast, there are multiple input voltages (typically 3 ) corresponding to each excitation force amplitude. 


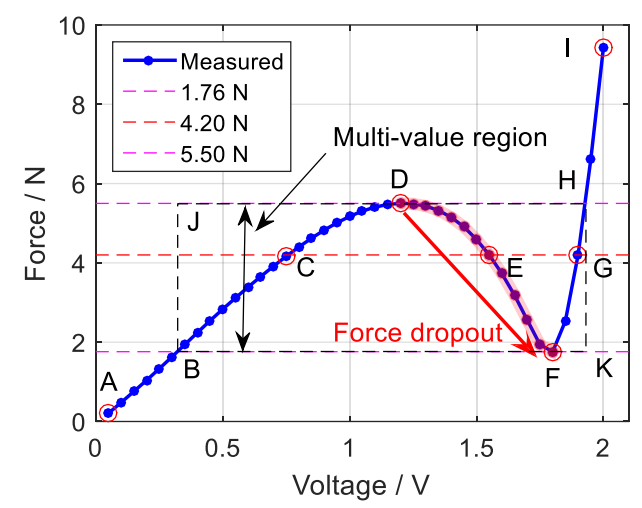

Fig. 11 The force drop-out phenomenon in the fixed frequency tests

The relationship between the first harmonic of the response amplitude, corresponding to the force input given in Fig. 11, and the voltage is shown in Fig. 12. The response amplitude is a monotonous and single valued function of the input voltage. Thus, there is a unique response amplitude corresponding to each voltage. The results from Figs. 11 and 12 show that, for the fixed frequency tests, there is a unique excitation amplitude and a unique response amplitude corresponding to each voltage. This indicates that the input voltage of the electromagnetic shaker can be used as a continuation parameter for the measurement of the multivalued response curves of strongly nonlinear systems. Generally speaking, one continuation parameter is required to guide the response curve through the turning point smoothly. As we discussed in Section 1, common continuation parameters, such as the arc length and pseudo arc length, can be used in numerical analysis or experiments. However, these parameters only have a geometrical rather than a physical meaning and cannot be controlled directly in experiments. Here we have exploited the force drop-out phenomenon and used the input voltage of the electrodynamic shaker as a continuation parameter to measure the multivalued response curve of a strongly nonlinear system in experiments. This is one of the first experiments to demonstrate the use of a continuation parameter with a physical meaning in practice. However, further research is still required to determine how far this behavior generalizes to other systems. A shaker-structure model is being developed to explain these phenomena through simulation, and hence to optimize the shaker characteristics, and the results will be reported in future research papers. 


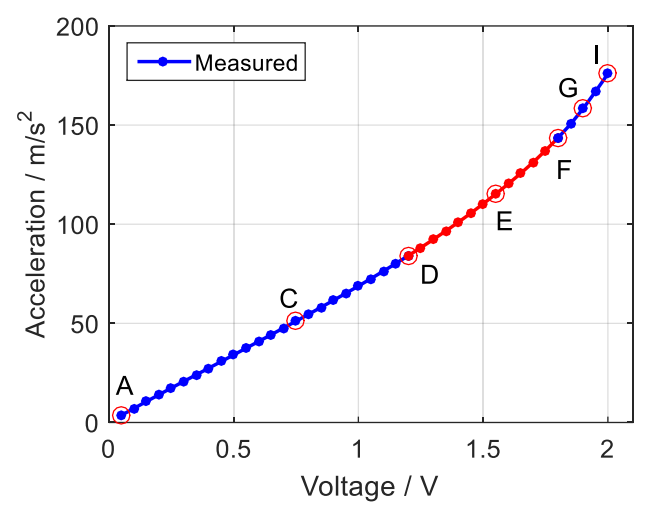

Fig. 12 The monotonic increase in the response amplitude with the input voltage

\section{Measuring the multivalued response curves at fixed frequencies}

\subsection{Measurement method}

In the theoretical analysis of a strongly nonlinear system, if there is a multivalued relationship between the excitation force amplitude, $F$, and the response amplitude, $R$, then the forceresponse curve may be written as an implicit function when the excitation frequency, $\omega$, is fixed. Thus

$$
f(F, R)=0
$$

This curve can be parameterized by a parameter, $\gamma$, which generally has a geometrical rather than a physical meaning. Then,

$$
\left\{\begin{array}{l}
F=F(\gamma) \\
R=R(\gamma)
\end{array}\right.
$$

Section 4 showed that the input voltage, $V$, of the electromagnetic shaker can be used as a natural continuation parameter. So when the excitation frequency is fixed, the excitation force amplitude and the response amplitude can be measured as a function the input voltage, that is

$$
\left\{\begin{array}{l}
F=F(V) \\
R=R(V)
\end{array}\right.
$$

The excitation force amplitude and the response amplitude are both single-value functions of the input voltage, and hence there is a unique excitation amplitude and response amplitude corresponding to each input voltage. Therefore, the relationship between excitation force 
amplitude and the response amplitude can be obtained directly and the multivalued response curve at this frequency can be established. This method exploits the force drop-out phenomenon of the electromagnetic shaker in fixed frequency tests and controls the variation of excitation amplitude automatically with the input voltage. The measurement process is shown diagrammatically in Fig. 13, and may be summarized as follows:

- Perform some preliminary vibration tests for the nonlinear structure, such as swept frequency sine tests, to obtain the variation range of the resonance frequencies in the frequency range of interest.

- Determine the input voltage range of the electromagnetic shaker for the fixed frequency tests. The excitation force should not be too large near resonance, to avoid damage to the structure.

- Perform fixed frequency tests and establish the curves of excitation amplitude with input voltage and response amplitude with input voltage for each frequency. If the excitation force amplitude falls with the input voltage, it indicates that there is multivalued response at this frequency; otherwise, there is no multivalued response.

- Finally, establish the multivalued response curves of strongly nonlinear systems from the measurements for the required range of fixed frequencies.

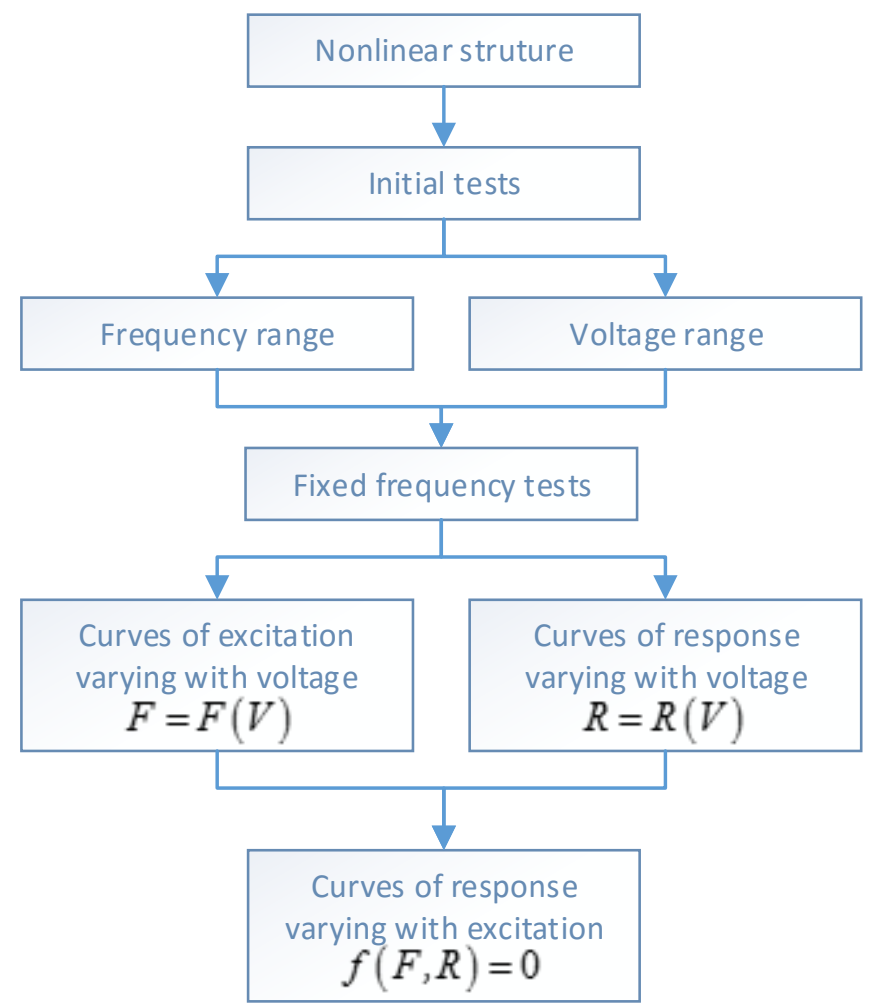

Fig. 13 The flowchart to measure multivalued response curves 


\subsection{Measured results}

The multivalued response curve obtained at $61.2 \mathrm{~Hz}$, for the example in Section 3.2, is shown as Fig. 14. The variation of the response amplitude with the excitation amplitude can be divided into three stages that correspond with those given in Section 4. Between points A and D, the excitation amplitude increases, and reaches the maximum at point $\mathrm{D}$. At the same time, the response amplitude also increases. From point $\mathrm{D}$ to point $\mathrm{F}$, the force drop-out occurs and the excitation force amplitude begins to decrease and reaches the minimum at F. During this process, the response amplitude still increases. After point F, the excitation amplitude begins to increase again, and the response amplitude also increases.

Because of the force drop-out in the second stage, a multivalued region is formed in the rectangular region between the line FB (about $1.76 \mathrm{~N}$ ) and HD (about $5.50 \mathrm{~N}$ ). In this multivalued region, there are multiple response amplitudes (typically 3 ) corresponding to the same excitation amplitude. For example, there are 3 response $\mathrm{C}, \mathrm{E}, \mathrm{G}$ when the force is $4.2 \mathrm{~N}$.

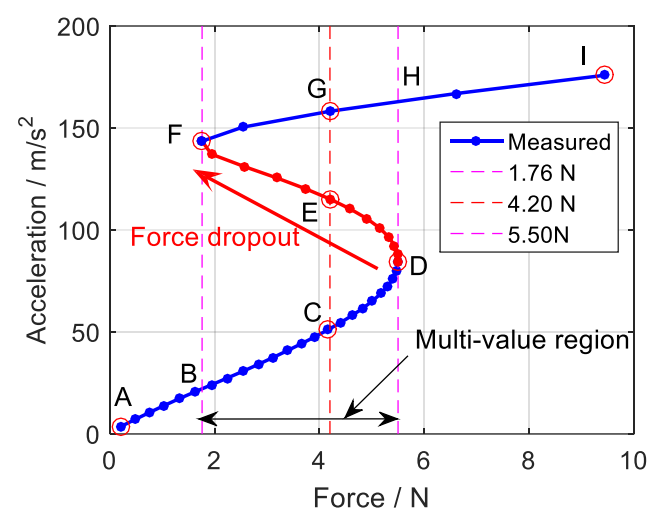

Fig. 14 The multivalued response curve at $61.2 \mathrm{~Hz}$

The multivalued response curves in the frequency range $59 \mathrm{~Hz}$ to $64 \mathrm{~Hz}$ with a step size of $0.1 \mathrm{~Hz}$ are shown in Fig. 15. The results at some frequencies, such as $61.5 \mathrm{~Hz}$ (the green line in Fig. 15. (b)), are incomplete. This is because the voltage range is the same at each frequency and since the force reduces significantly at resonance, the resulting excitation is too small. To obtain the complete multivalued response curve at this frequency, the voltage range should be increased, although at resonance this may risk permanent damage to the structure. Once multivalued response curves from fixed frequency tests are obtained, the required response surface and frequency response functions could be created using Gaussian process regression [29]. 


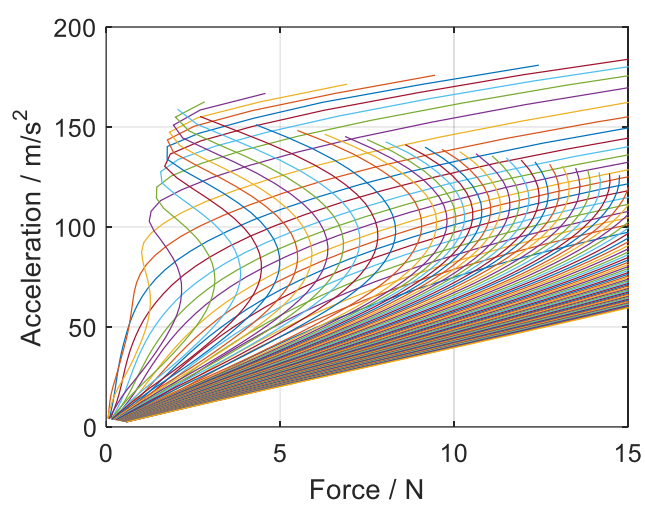

(a) Response curves at different frequencies

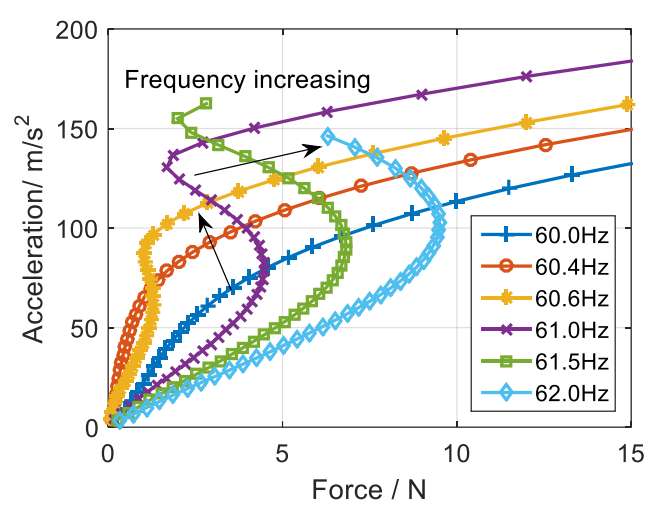

(b) Response curves at frequencies near resonance

Fig. 15 The multivalued response curves from $59 \mathrm{~Hz}$ to $64 \mathrm{~Hz}$

\section{Characteristics of the multivalued responses and the influence of}

\section{excitation}

The characteristics of the multivalued responses and the influence of excitation are analyzed in this section, taking the test data of the strongly nonlinear system described in Section 3.2 at $61.2 \mathrm{~Hz}$ as an example.

The time domain test data at the points C, E, and G in Fig. 14 are shown in Fig. 16. Initially no force is applied to the system for $0.1 \mathrm{~s}$, and it can be clearly seen that each test begins with zero initial conditions. Starting from zero initial conditions is possible in this case because of the single valued nature of the force and response amplitudes as a function of input voltage. Standard stepped or swept sine tests that vary excitation frequency or force amplitude will often contain jumps and hence require only small transients by ensuring the continuity of the force excitation. The sampling time is sufficiently long for the excitation force and the response to reach steady state. A stability assessment of the solutions is necessary in experiments in order to improve efficiency, and there are several methods to assess stability in experiments [29][32], which could be added to the fixed frequency tests in future. The three chosen voltage levels have the same force amplitude at the excitation frequency, however the time history of excitation at the three voltage levels are not the same. The difference arises because of the presence of harmonics in the excitation force which will be seen in the frequency domain analysis later. The response amplitudes of increase gradually from $\mathrm{C}$ to $\mathrm{E}$ to $\mathrm{G}$.

An interesting fact is that the measured response at point $\mathrm{E}$ on the unstable branch has reached a stable steady state under zero initial conditions. Normally this solution is considered as unstable and is not observed in practice. In this case the complete system, composed of the 
nonlinear structure and the exciter, is indeed stable and so the steady state is achieved in the experiment [14].

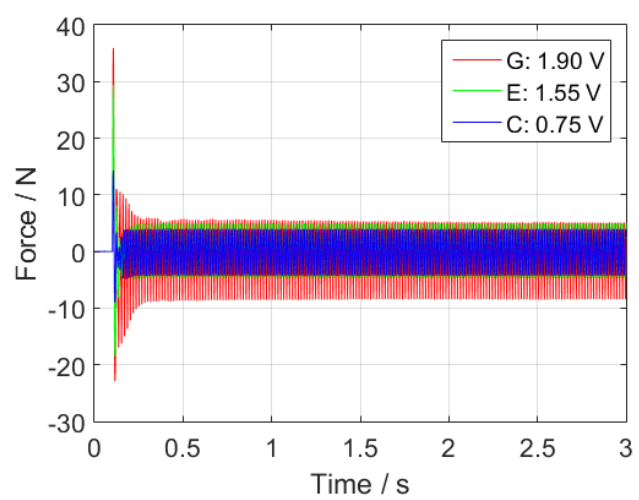

(a) Time-history of excitation

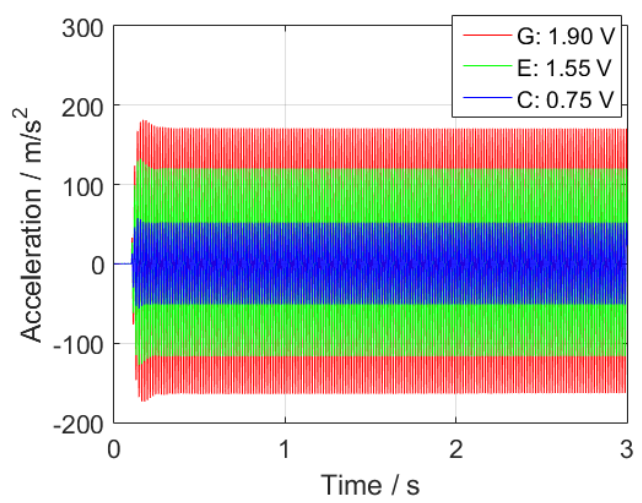

(b) Time-history of response

Fig. 16 The measured time domain data for the strongly nonlinear system

In order to further analyze the multivalued phenomenon, Fig. 17 shows the steady-state time domain data at points $\mathrm{C}, \mathrm{E}$, and $\mathrm{G}$, as well as their order spectrum obtained from the FFT. In the time domain, the force at point $\mathrm{C}$ is almost harmonic, but the steady state excitation becomes more distorted at higher input voltage levels. Higher order harmonics, especially the second and third harmonics, are often found. These harmonics arise because of the nonlinearity in the structure, and possibly also in the shaker, and the coupling between the shaker and the structure leads to nonlinear behavior and harmonics in the excitation force at large amplitudes of vibration [15][20]. Notice that the amplitudes of the first harmonic at points C, E and G are identical and $4.2 \mathrm{~N}$, but the amplitudes of the higher order harmonics, especially the second and third harmonics, are very different. The presence of the higher harmonics is the reason for the distortion in the time domain force signals. 

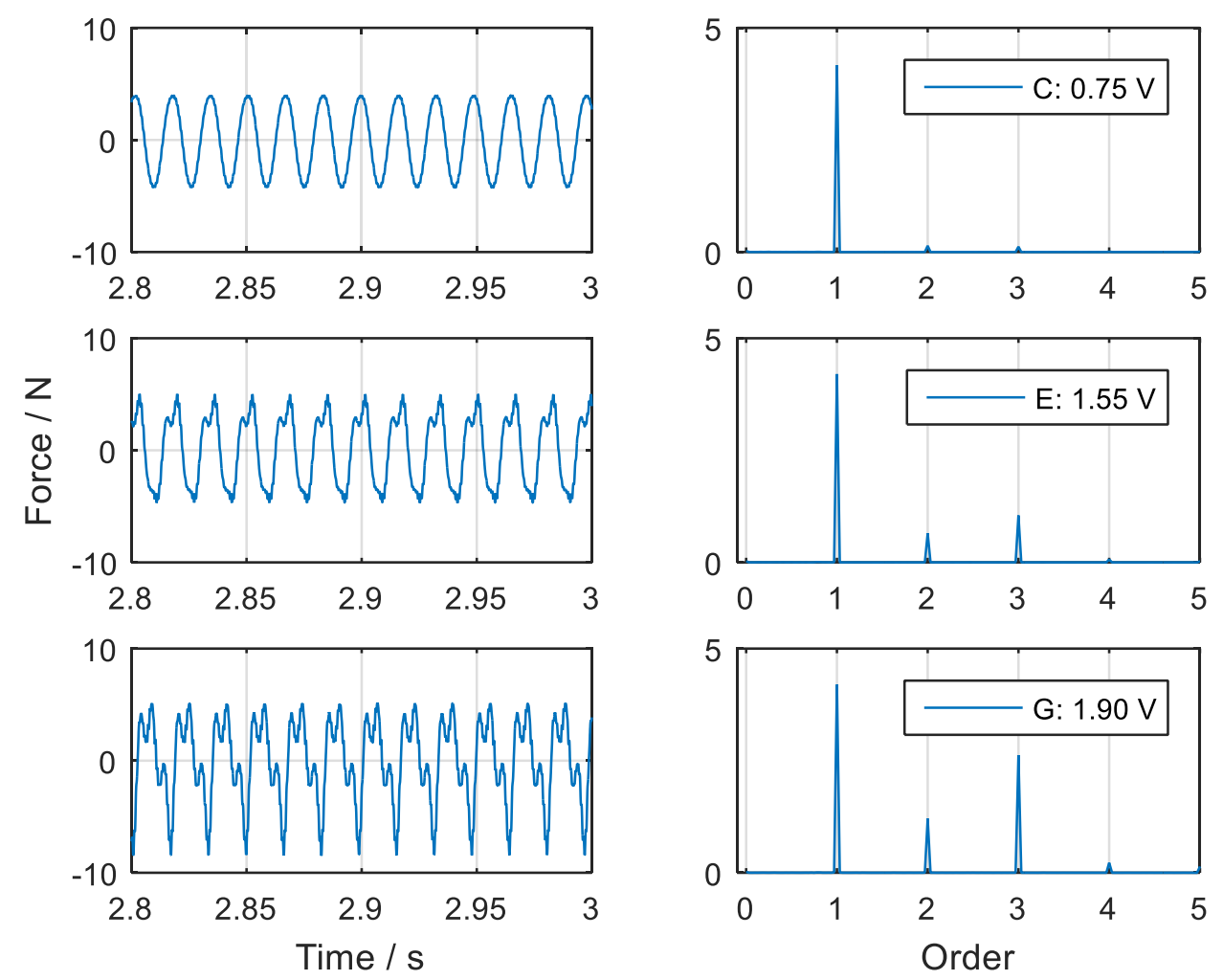

(a) Time history

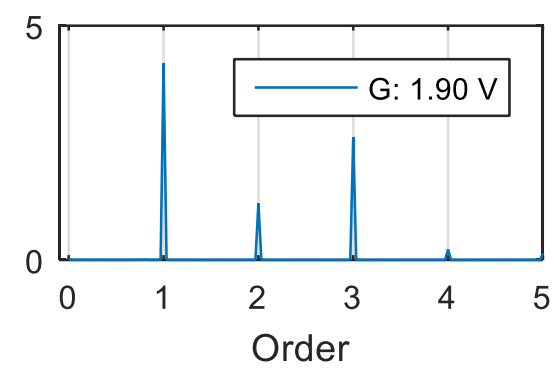

(b) Order spectrum

Fig. 17 The steady state force excitations at different input voltage levels and their order spectra

The steady-state response data and their order spectra at points C, E, and G are shown in Fig. 18. Although the higher order harmonics appear in the response, their amplitudes are small and the harmonic distortions in the time response are not obvious. 


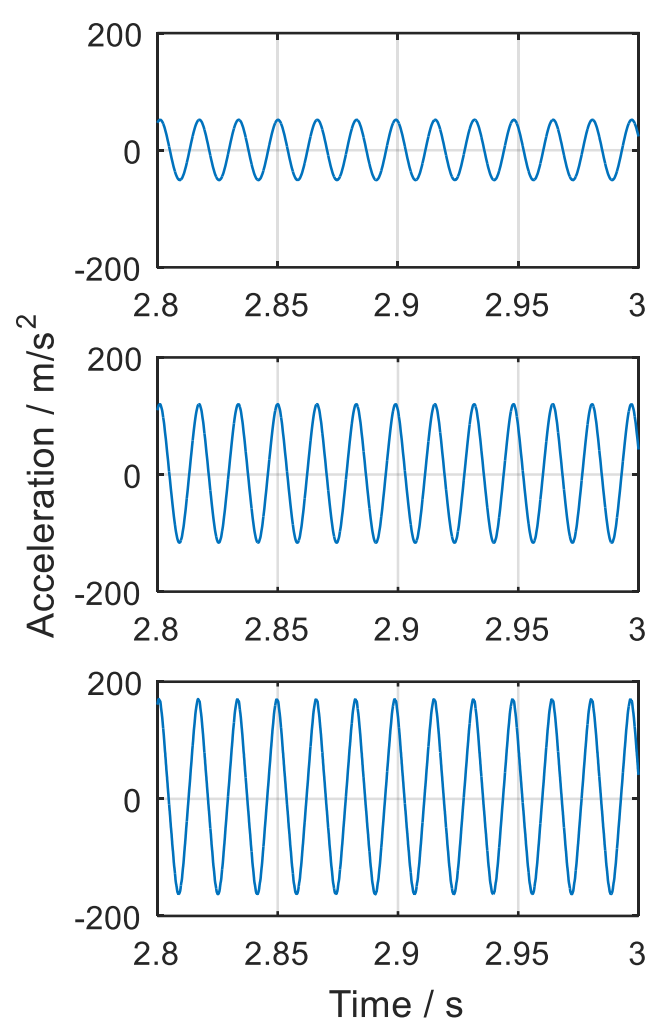

(a) Time history
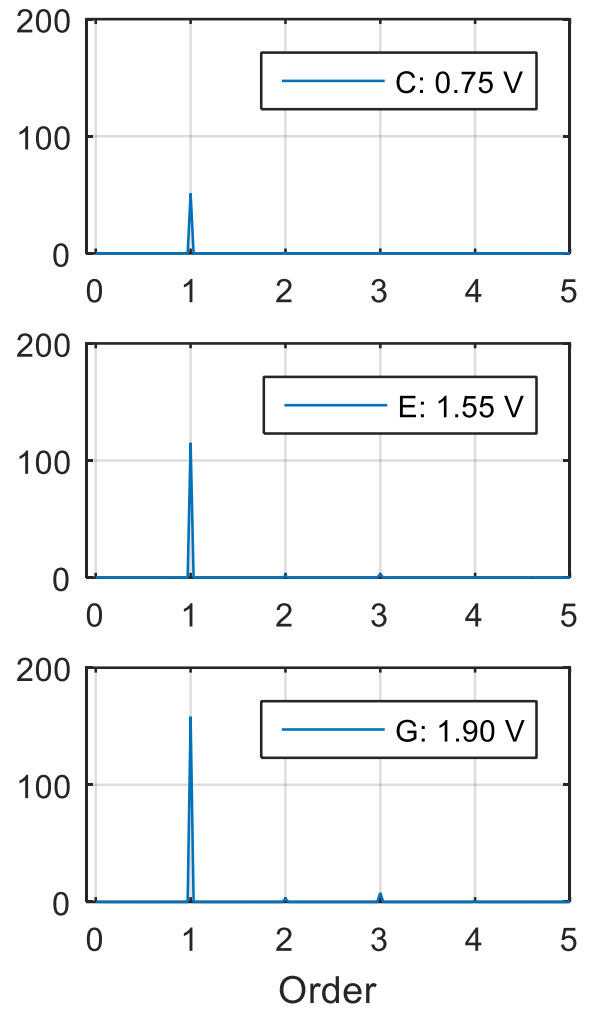

(b) Order spectrum

\section{Fig. 18 The steady state responses at different input voltage levels and their order}

spectra

Figure 17 shows that the excitation force does contain higher order harmonics. Their influence on the response is now analyzed more comprehensively. The relationship between the amplitudes of the first three harmonics of the excitation force and the input voltage is shown in Fig. 19. The amplitude of the first harmonic clearly shows the force drop-out, which has already been discussed extensively. However, the amplitudes of the second and third harmonics increase monotonically and reflects the increase in the response. Near resonance, the amplitude of the first harmonic could be smaller than the amplitude of the third harmonic.

The relationship between the amplitudes of the first three harmonics of the responses and the input voltage is shown in Fig. 20. Clearly, with the increase of the input voltage, the amplitudes of all three harmonics also increase. The amplitude of first harmonic still dominates in the response and the amplitudes of other two harmonics are obviously much smaller. These harmonic components also maintain a monotonically increasing trend. 


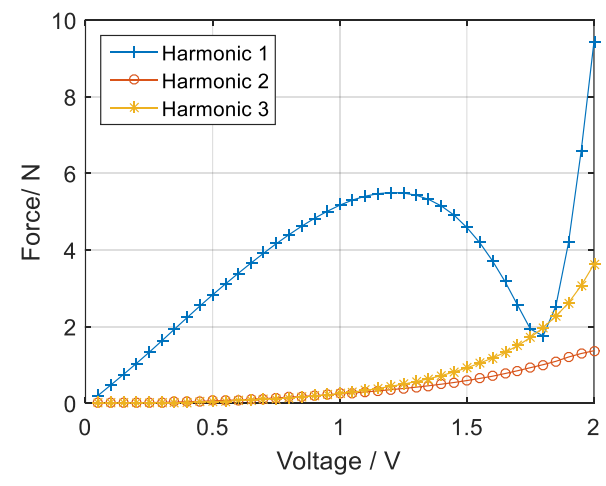

Fig. 19 The main harmonics of the excitation force as a function of the input voltage

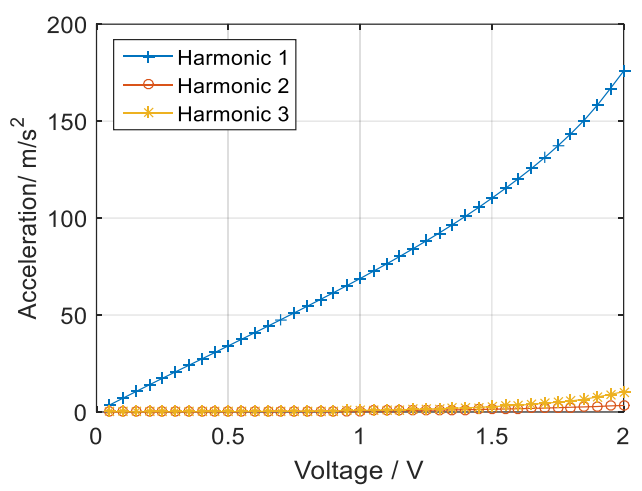

Fig. 20 The main harmonics of the response amplitude as a function of the input voltage

The relationship between the first harmonic of the excitation and the response is given in Fig. 14 and shows the multivalued response curve. The curves of the second and third harmonic of the excitation and with the corresponding harmonic of the response are shown in Fig. 21 and Fig. 22, respectively. The relationship between the second harmonic (and the third harmonic) of the excitation and response is basically linear. Overall, the higher order harmonics of the excitation force are likely to have only a limited effect on the first harmonic of the response for this nonlinear system. Strictly speaking, high order harmonics in the force should be eliminated, to replicate the simulated tests and analysis. If necessary, a control system may be used to eliminate the higher harmonics in the excitation force, by adding harmonics into the input voltage signals. This has been done for standard stepped sine testing with a constant force amplitude [33]. The same approach could be adopted here, with the slight difference that the amplitude of the fundamental component of the input voltage is fixed rather than the fundamental component of the excitation force. This approach will be studied in future work. 


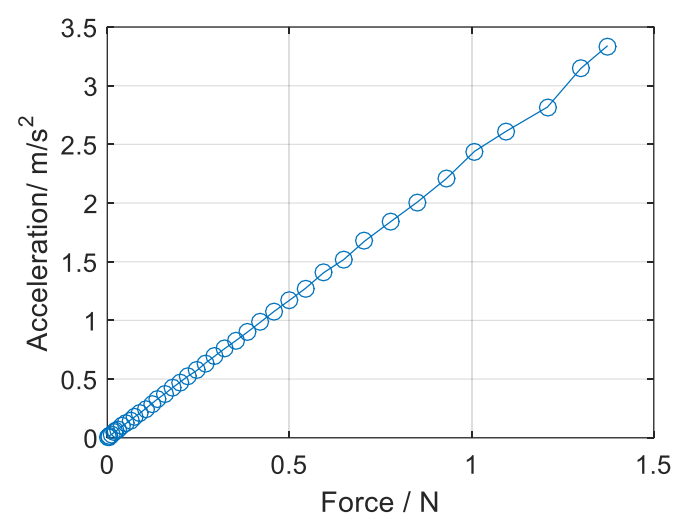

Fig. 21 The second harmonic of the response as a function of the second harmonic of the excitation force

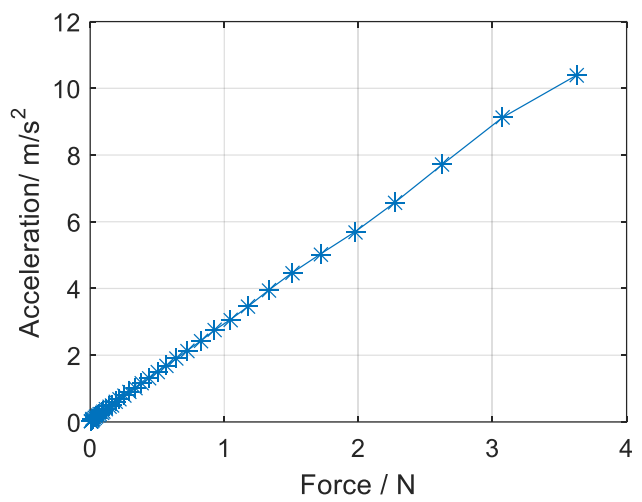

Fig. 22 The third harmonic of the response as a function of the third harmonic of the excitation force

\section{Conclusions}

In this paper, the force drop-out phenomenon in fixed frequency tests is exploited in the experimental testing of strongly nonlinear systems. The input voltage of the electromagnetic shaker is used as a natural continuation parameter in the fixed frequency tests. Based on these two findings, an experimental measurement method for multivalued response curves for strongly nonlinear systems is proposed. This method uses the force drop-out phenomenon of electromagnetic shaker in fixed frequency tests to automatically and passively control the variation of excitation amplitude. With the input voltage as the continuation parameter the multivalued response curves through the force drop-out are measured. The coupling with the exciter increases the damping in the system and stabilizes the unstable branch of the strongly nonlinear system in isolation. This method is very simple and easy to perform without any need of feedback control. 
The objectives of this paper are to propose a new approach to testing nonlinear structures and to demonstrate that it works on a relatively simple structure. Significant further research is required to develop and optimize the testing procedure and some of these research directions are now summarized. The input voltage of the electromagnetic shaker is increased with a constant step size. Although the multivalued response curves can be measured, the information at the turning point cannot be obtained accurately, which is a disadvantage for experimental bifurcation research. In order to improve the test efficiency and to accurately obtain the information at the turning point, a variable step size control can be used. Methods to assess the stability of the solutions may also be added to fixed frequency tests. The structure-shaker interaction should be modelled to determine the conditions for the stability of the assembled system and to optimize the shaker characteristics. Current methods to remove the higher order harmonics from the excitation during the experiments need to be extended to the voltage controlled approach described in this paper. In addition, the proposed method has only been demonstrated on a SDOF system with cubic nonlinearity; the method needs to be further verified and improved for the application to other nonlinear structures.

\section{Acknowledgments}

The authors gratefully appreciate the financial support for this work provided by the National Natural Science Foundation of China and National Safety Academic Foundation of China (No. U1730129), The Cultivation Foundation of National Defense Large Projects of China (No. NP2018450), Jiangsu Innovation Program for Graduate Education (Project No. CXLX13_167), the Fundamental Research Funds for the Central Universities, Funding for Outstanding Doctoral Dissertation in NUAA (Project No. BCXJ13-04). The support of the Jiangsu Province Key Laboratory of Aerospace Power System, the Key Laboratory of Aero-engine Thermal Environment and Structure, Ministry of Industry and Information Technology are also gratefully acknowledged.

\section{References}

[1] G. Kerschen, K. Worden, A.F. Vakakis, J.-C. Golinval, Past, present and future of nonlinear system identification in structural dynamics. Mechanical Systems and Signal Processing, 2006, 20(3): $505-$ 592.

[2] A.H. Nayfeh, D.T. Mook, Nonlinear oscillations. John Wiley \& Sons, 1979.

[3] D.J. Wagg, S.A. Neild, Nonlinear vibration with control. Springer-Verlag, 2009.

[4] X. Wang X, T.L. Hill, S.A. Neild, A.D. Shaw, H. Haddad Khodaparast, M.I. Friswell, Model updating strategy for structures with localised nonlinearities using frequency response measurements. Mechanical Systems and Signal Processing, 2018, 100: 940-961. 
[5] E.P. Petrov, Frequency-domain sensitivity analysis of stability of nonlinear vibrations for highfidelity models of jointed structures. Journal of Engineering for Gas Turbines and Power, 2018, 140(1): 012508 .

[6] R. Lacayo, L. Pesaresi, J. Groß, D. Fochler, J. Armand, L. Salles, C. Schwingshackl, M. Allen, M. Brake, Nonlinear modeling of structures with bolted joints: A comparison of two approaches based on a time-domain and frequency-domain solver. Mechanical Systems and Signal Processing, 2019, 114: 413-438.

[7] J.P. Noël, L. Renson, G. Kerschen, Complex dynamics of a nonlinear aerospace structure: experimental identification and modal interactions. Journal of Sound and Vibration, 2014, 333(12): 2588-2607.

[8] F. Braghin, F. Resta, E. Leo, G. Spinola, Nonlinear dynamics of vibrating MEMS. Sensors and Actuators A: Physical, 2007, 134(1): 98-108.

[9] S.B. Cooper, D Di Maio, D.J. Ewins, Integration of system identification and finite element modelling of nonlinear vibrating structures. Mechanical Systems and Signal Processing, 2018, 102: 401-430

[10] S. Stoykov, S. Margenov, Numerical computation of periodic responses of nonlinear large-scale systems by shooting method. Computers \& Mathematics with Applications, 2014, 67(12): $2257-$ 2267.

[11] G. von Groll, D.J. Ewins, The harmonic balance method with arc-length continuation in rotor/stator contact problems. Journal of sound and vibration, 2001, 241(2): 223-233.

[12] R. Seydel, Practical bifurcation and stability analysis. Springer Science \& Business Media, 2009:169-196.

[13] T. Detroux, L. Renson, L. Masset, G. Kerschen, The harmonic balance method for bifurcation analysis of large-scale nonlinear mechanical systems. Computer Methods in Applied Mechanics and Engineering, 2015, 296: 18-38.

[14] J.V. Ferreira, Dynamic response analysis of structures with nonlinear components. PhD Thesis, Imperial College London (University of London), 1998.

[15] S. Perinpanayagam, D. Robb D, D.J. Ewins, et al., Non-linearities in an aero-engine structure: from test to design. Proceedings of the 2005 International Conference on Modal Analysis, Noise and Vibration Engineering, Leuven, Belgium. 2004: 3167-3182.

[16] K. Worden, G.R. Tomlinson, Nonlinearity in structural dynamics: detection, identification, and modeling, 2001: Taylor \& Francis.

[17] G.R. Tomlinson, Force distortion in resonance testing of structures with electro-dynamic vibration exciters. Journal of Sound and Vibration, 1979, 63(3): 337-350.

[18] N.L. Olsen, Using and understanding electrodynamic shakers in modal applications. Proceedings of the Forth International Modal Analysis Conference, Los Angeles, California, USA. 1986: 3-6.

[19] D. Rao, Electrodynamic interaction between a resonating structure and an exciter. The 5th International Modal Analysis Conference, London, England. 1987: 1142-1150.

[20] G.R. Tomlinson, A simple theoretical and experimental study of the force characteristics from electro dynamic exciters on linear and nonlinear systems. Proceedings of the 5th International Modal Analysis Conference, 1987.

[21] S. Huang, Dynamic analysis of assembled structures with nonlinearity. PhD Thesis, Department of Mechanical Engineering, Imperial College London, 2008.

[22] A. Haddow, Testing, Nonlinear System. Encyclopedia of Vibration, 2001: 1285-1289. 
https://doi.org/10.1006/rwvb.2001.0040.

[23] J.V. Ferreira, A.L. Serpa, A.P. Prado, Experimental nonlinear frequency response determination using the arc-length method. IMAC-XXI: Conference \& Exposition on Structural Dynamics. 2003.

[24] J. Sieber, B. Krauskopf, Control based bifurcation analysis for experiments. Nonlinear Dynamics, 2008, 51(3): 365-377.

[25] J. Sieber, B. Krauskopf, D.J. Wagg, S.A. Neild, A. Gonzalez-Buelga, Control-based continuation of unstable periodic orbits. Journal of Computational and Nonlinear Dynamics, 2011, 6(1): 011005.

[26] L. Renson L, D.A.W. Barton, S.A. Neild, Experimental tracking of limit-point bifurcations and backbone curves using control-based continuation. International Journal of Bifurcation and Chaos, 2017, 27(01): 1730002 .

[27] D.A.W. Barton, B.P. Mann, S.G. Burrow, Control-based continuation for investigating nonlinear experiments. Journal of Vibration and Control, 2012, 18(4): 509-520.

[28] D.A.W. Barton, J. Sieber, Systematic experimental exploration of bifurcations with noninvasive control. Physical Review E, 2013, 87(5): 052916.

[29] D.A.W. Barton, Control-based continuation: Bifurcation and stability analysis for physical experiments. Mechanical Systems and Signal Processing, 2017, 84: 54-64.

[30] S. Peter, M. Scheel, M. Krack, R.I. Leine, Synthesis of nonlinear frequency responses with experimentally extracted nonlinear modes. Mechanical Systems and Signal Processing, 2018, 101: 498-515.

[31] G.B. Zhang, C.P. Zang, M.I. Friswell, Identification of weak nonlinearities in MDOF systems based on reconstructed constant response tests. Archive of Applied Mechanics, 2019, 89: 2053-2074.

[32] E. Bureau, F. Schilder, M. Elmegård, Experimental bifurcation analysis of an impact oscillatorDetermining stability. Journal of Sound and Vibration, 2014, 333(21): 5464-5474.

[33] A.D. Shaw, T.L. Hill, S.A. Neild, M.I. Friswell, Periodic responses of a structure with 3: 1 internal resonance. Mechanical Systems and Signal Processing, 2016, 81: 19-34. 